\title{
Gestión del proceso de capacitación en las áreas soporte de negocio en una empresa contratista de minería y construcción en Lima
}

\author{
Elizabeth del Carmen Velásquez Fuentes \\ elizabethdelcarmenvelasquezfue@gmail.com \\ ORCID: 0000-0003-2235-1904 \\ Universidad César Vallejo - Lima Perú
}

\section{RESUMEN}

El presente estudio tuvo como objetivo realizar un diagnóstico de la forma como se ejecuta actualmente el proceso de capacitación dirigido a las áreas de soporte del negocio de la empresa minera SMCG y en base a este diagnóstico se elaboró una propuesta de mejora. El enfoque de estudio es cualitativo y de naturaleza descriptiva, asimismo se desarrolló una propuesta de mejora bajo un proceso de capacitación, se identificaron las necesidades de capacitación en el personal de la empresa de construcción y minería de Lima, con el fin de promover actividades educativas que permitan alcanzar los objetivos y metas, relacionados con la política de calidad y demás políticas organizacionales. Los resultados determinaron que el desarrollo de las competencias en la empresa de construcción y minería de Lima es fundamental en el marco de un enfoque estratégico, pues hay que hacer evolucionar las competencias de la empresa y de sus colaboradores en función de las orientaciones estratégicas. Esto implica una orientación a la formación permanente de sus recursos humanos a través de la capacitación.

Se concluye, indicando que la empresa Contratista en Minería y Construcción de Lima, es una de las compañías que aplica herramientas y estrategias modernas de gestión en todos sus procesos organizacionales, por otro lado, la política de Gestión de Recursos Humanos se encuentra articulada de la Gerencia General, siendo los líderes y fundadores los que plantean dicha política.

Palabras clave: capacitación; diagnóstico; propuesta de mejora; áreas de soporte; capital humano 


\title{
Management of the training process in the business support areas in a mining and construction contractor company in Lim
}

\begin{abstract}
The objective of this study was to carry out a diagnosis of the way in which the training process aimed at the business support areas of the mining company SMCG is currently being carried out, and based on this diagnosis, an improvement proposal was prepared. The study approach is qualitative and descriptive in nature, an improvement proposal was also developed under a training process, the training needs of the personnel of the Lima construction and mining company were identified, in order to promote educational activities that allow to achieve the objectives and goals, related to the quality policy and other organizational policies. The results determined that the development of competencies in the Lima construction and mining company is essential within the framework of a strategic approach, since the competencies of the company and its collaborators must be made to evolve according to the strategic orientations. This implies an orientation to the permanent formation of its human resources through training.

It concludes, indicating that the company Contractor in Mining and Construction of Lima, is one of the companies that applies modern management tools and strategies in all its organizational processes, on the other hand, the Human Resources Management policy is articulated from the General Management, being the leaders and founders who propose said policy.
\end{abstract}

Keywords: training; diagnosis; improvement proposal; support areas; human capital

Artículo recibido: 30 noviembre. 2021

Aceptado para publicación: 29 diciembre 2021

Correspondencia: elizabethdelcarmenvelasquezfue@gmail.com

Conflictos de Interés: Ninguna que declarar 


\section{INTRODUCCIÓN}

El desarrollo de la presente investigación "Análisis de la gestión del proceso de capacitación en las áreas soporte de negocio en una empresa contratista de minería y construcción en Lima" tuvo como objetivo general desarrollar una Propuesta de Capacitación y Evaluación de Personal en una de las empresas líderes en Contratista en Minería y Construcción de Lima, con más de 10 años en el mercado de la construcción. Se analizó esta empresa, debido a la complejidad de su sistema organizacional, con una planilla aproximadamente de doscientos trabajadores entre administrativos y operarios, se debe contar con las herramientas, técnicas, estrategias en Recursos Humanos (RRHH) y conocimientos necesarios para poder manejar tan grande organización.

Tradicionalmente las empresas consideran a su plantilla como un costo social que hay que reducir al mínimo. Por otro lado, está la tendencia actual donde las empresas ven en su personal un recurso humano en los que ellas invierten. La diferencia fundamental está que la segunda concepción, a diferencia de la primera, considera a la función social como fundamental en el incremento de la competitividad de la empresa.

¿Pero a qué se debe la creciente importancia de los recursos humanos? Las organizaciones se están volviendo más dependientes de las personas, porque están cada vez más relacionadas con tecnologías más complejas y están tratando de funcionar en ambientes económicos, políticos, y socioculturales más complejos. De modo que, cuanto más intervienen diferentes destrezas técnicas en el proceso de diseño, fabricación, comercialización de un producto, más vulnerable será la organización sino cuenta con recursos humanos adecuados.

Entonces, se menciona que un sistema básico de gestión de recursos humanos es aquel que incluye un planeamiento tanto individual como organizacional, y una serie de actividades de adecuación diseñadas para necesidades mutuas. Si además asumimos que tanto las necesidades individuales como las organizacionales cambian con el tiempo, este sistema debe considerar el planeamiento para las designaciones de cargos, desarrollo, nivelación y desenganche y reemplazo o redesignación. El objetivo es asegurar que la organización tenga una base adecuada para seleccionar sus recursos humanos y desarrollarlos hacia el cumplimiento de las metas organizacionales. La capacitación es sin duda el instrumento central en la nueva práctica de la gestión de recursos humanos. 


\section{Gestión del Talento Humano}

El estudio acerca de la gestión del talento humano viene cobrado amplio interés en la literatura de los recursos humanos; en particular, como un aspecto vital que contribuye al desarrollo de redes de conocimiento e innovación dentro de las organizaciones empresariales. En este contexto, es necesario conocer y analizar los ejes determinantes que inciden en el funcionamiento de los recursos humanos. Viltard (2016) señaló que "los activos físicos ya no producen el crecimiento que las organizaciones esperan; el capital intelectual se ha convertido en el determinante supremo de la viabilidad de los negocios presentes y su sostenibilidad en el futuro" (p.13). Bajo el enfoque de Viltard se hace conducente tener de manera clara la importancia que tienen los colaboradores en las organizaciones.

Desde una perspectiva global, el CIPD (Chartered Institute of Personnel and Development, Reino Unido) señala y analiza la aplicación de la teoría de recursos y capacidades al campo de los recursos humanos. Se afirmó mediante este enfoque que podía constituirse en una fuente de ventaja competitiva (Cappelli, P; Singh, U, 2014). Consecutivamente se diseñaron los fundamentos para la denominada gestión estratégica de recursos humanos. Así, se acentuó la jerarquía del talento humano, en la procreación de utilidades a partir de la creación de habilidades específicas a la empresa (Ospina, 2010).

En la línea de formación de un recurso estratégico y apto para crear prerrogativas competitivas sostenibles en el tiempo son: ser valioso, poco común, no imitable y no canjeable, es decir, que agreguen valor y que exista un mercado para conseguirlos, por lo tanto, la organización además de atraer y retener talentos debe establecer mecanismos que contribuyan a maximizar su contribución al valor agregado (Barney, 2015).

Chiavenato (2011) señaló que estamos ante un talento cuando el colaborador se convierte en un recurso estratégico para la empresa. Define al talento como cualquier recurso que la persona tenga (conocimientos, capacidades, características personales, red de contactos, etc.) que hace que su persona sea generadora de valor. Se añade que "el talento es inimitable, intangible y uno de los ejes de las ventajas competitivas de las empresas. Cambia su estatus de variable blanda a variable dura sobre los resultados.” (pág., 13) Como se puede apreciar, el talento humano se asienta bajo los criterios de valor que aportan a la organización y a la especificidad de sus conocimientos, habilidades y 
destrezas y que por tanto no están a disposición de un elevado número de empresas. Atchison, (1991); Osterman, (1994); Prahalad y Hamel, (1990) señalaron que estas características se estudian en los trabajadores centrales de la organización, es decir, en el conjunto de colaboradores que definen el proceso clave y se encuentra vinculados con el desarrollo de las capacidades centrales o nucleares de la empresa, exceptuando a la dirección de la misma (López \& Valle, 2017). López-Cabrales señalaron que este colectivo es considerado el verdadero recurso humano estratégico, es decir, el capital humano que debe ser retenido y fidelizado (Ojeda, 2018).

De lo anterior se pueden identificar que los talento o altos potenciales dependerá de la estrategia de la organización y los parámetros que ésta ha establecido para diferenciarlos. A continuación, se presentan las principales razones propuestas por Deloitte Consulting (2014), las empresas deberían tomar en cuenta para invertir en la gestión del talento:

- Motivación de los empleados: crear más razones para que los empleados se sientan atraídos por la organización, como un propósito o un significado superior para los empleados.

- Atraiga al mejor talento: Se sugiere reclutar empleados más talentosos y capacitados disponibles. Cuando tiene una gestión estratégica del talento, puede crear una marca de empleador, que atrae orgánicamente a su talento ideal y, a su vez, contribuye a niveles más altos de desempeño y resultados comerciales.

- Cobertura continua de roles críticos: una organización deberá estar preparada para llenar vacíos en habilidades críticas y deberá tendrá un plan para abordar los roles críticos y los roles altamente especializados en la fuerza de trabajo. Esto significa que una organización tendrá un flujo continuo de empleados para cumplir roles críticos, lo que garantiza que las operaciones se desarrollen sin inconvenientes y que sus clientes y partes interesadas estén satisfechos. También significa que otros empleados no se quedan con cargas de trabajo adicionales, lo que eventualmente podría llevar al agotamiento.

- Aumente el rendimiento de los empleados: es más fácil identificar a los empleados con buena adaptación, en lugar de tomar decisiones en la contratación que no funcionan para la estrategia organizativa ideal. Esto puede conducir a problemas y reclamos de gestión del rendimiento. También asegurará que los mejores talentos dentro de la organización permanezcan más tiempo. 
- Empleados comprometidos: una organización puede tomar decisiones sistemáticas y consistentes sobre el desarrollo del personal, asegurando que las personas que usted lo requiera tengan las habilidades y el desarrollo necesarios, y ahorren dinero en desarrollo innecesario. Además, cuando hay un proceso justo para el desarrollo, los empleados se sienten más comprometidos y esto nuevamente aumenta las tasas de retención y también asegura que la organización pueda cumplir con sus requisitos operativos.

- Retenga el mejor talento: las prácticas de incorporación bien estructuradas crean un alto porcentaje de más de niveles de retención. Esto significa que una organización ahorra en costos de administración de reclutamiento y rendimiento a largo plazo.

- Mejore el rendimiento comercial: cuando los empleados están comprometidos, capacitados y motivados, trabajarán para alcanzar sus objetivos comerciales, lo que a su vez aumentará la satisfacción del cliente y el desempeño comercial.

- Mayor satisfacción del cliente: un enfoque sistemático para la gestión del talento significa que existe una integración amplia de la organización y un enfoque coherente para la gestión. Esto a su vez se traduce en comunicación general y disolución de silos dentro del negocio. Cuando los sistemas están más integrados, las tasas de satisfacción del cliente suelen ser más altas, ya que se ocupan de menos personas y sus necesidades se satisfacen más rápidamente (Deloitte Consulting LLP \& Deloitte, 2014).

De acuerdo con las razones citadas, es evidente la importancia que tiene el recurso humano en el éxito de las empresas, lo que es ampliamente reconocida en la literatura. Viltard (2016) cita a Barney \& Wrigth (1998) señalando que "aspectos como las capacidades y competencias distintivas de las personas que laboran en la organización resultan cruciales para la competitividad empresarial, aspecto que ha sido subrayado desde el surgimiento de la teoría de recursos y capacidades" (p. 12).

Tomando el enfoque de Viltard, es preciso contrastarlo con lo señalado por Ospina (2010) quien indica que tradicionalmente la gestión humana se ha focalizado en el interior de la empresa, básicamente en preocuparse por las personas y sus necesidades y esta es una tarea que deberá continuar haciendo. Sin embargo, actualmente se le plantean nuevas exigencias: construir el área de afuera hacia adentro y diseñar la organización hacia el alto desempeño. En efecto, la gestión del desempeño alineada a la estrategia contribuye a que el área de gestión humana, lo que Ulrich (2013) denomina socio estratégico, ya que 
de esta manera las áreas de Recursos Humanos comprenden exactamente cuáles son los direccionadores estratégicos de la organización, su estrategia y objetivos fundamentales y cómo dicha área contribuye a la organización para alcanzarlos.

La relevancia de centrar la doctrina y lo que indican los autores acerca del talento humano es necesaria debido a la articulación que tiene con temas convexos y que son ejes principales para que el talento humano sea productivo. El tema central al que compete la investigación es la capacitación que se analizada también como entrenamiento y ello se perfila como una variable de competencia de los recursos humanos; es bajo esta idea que se ha centrado primero la base teórica de la gestión del talento humano. Sin embargo, se hace también indispensable tomar en cuenta el conocimiento, de cómo ello impacta en la buena gestión de los recursos humanos, Macías \& Martínez (2012) indican que "la popularidad de la gestión del conocimiento ha estado creciendo desde la mitad de los años noventa y se ha convertido desde entonces, en una filosofía y herramienta gerencial". De acuerdo con los enfoques que se vienen citando, la exploración del conocimiento del talento humano guarda íntima relación con el nivel que adquieran los colaboradores y mucho tiene que ver el tema de las capacitaciones.

Por otro lado, es necesario también indicar que en toda entidad el desarrollo de la cultura contribuye en el crecimiento de lo novedoso, permitiendo que las actividades se lleven de forma fácil y convenientes dejando generar conocimientos manejados por cada uno de los colaboradores (Ureña, Jimenez, Mejía, \& Vilarete Molina, 2014). En efecto, la capacitación se debe tener como un valor y objetivo estratégico de las organizaciones que apuntan en establecer un binomio compuesto por los recursos humanos y la generación de una rentabilidad cuidando el capital humano. Pérez, Prieto, \& Sierra (2014) sostuvieron que, si la obtención de buenos resultados de empleado depende de su habilidad, su motivación y la oportunidad para obtener esos resultados, entonces el logro de unos resultados en gestión del conocimiento radica en la creación y transferencia de conocimiento articulada a las habilidades, motivación y oportunidades de los empleados para crear y transferir conocimiento, desde luego tiene que ver mucho las capacitaciones.

\section{Concepto de la capacitación}

El ser humano es uno de los recursos más importantes para una empresa, actualmente las organizaciones vienen prestando mucha atención al desarrollo y promoción de talentos, creando una plataforma para el personal a través de la construcción de una organización 
de aprendizaje. Debiéndose apostar por un concepto orientado a los recursos humanos para alentar a cada persona a desafiar las capacidades individuales, motivando el entusiasmo del personal, para lograr construir una empresa de marca que permita acumular logros y obtener valor atrayendo, uniendo y cultivando talentos (EDWPUE, 2016). Se ha creído conveniente empezar haciendo referencia al aprendizaje en las organizaciones como elemento fundamental de la capacitación, toda vez, que el adiestramiento o entrenamiento tiene como eje principal el aprendizaje, operacionalmente el personal o recurso humano involucrado en cualquier proceso empresarial debe tener en mente el aprendizaje a lo largo de su línea de carrera; debido a que para consolidar una adecuada capacitación se hace necesario el aprendizaje.

En concordancia con lo indicado por la Consultoría colombiana EDWPUE es coherente con lo dicho por Cros (2016) que la "capacitación empresarial se ha convertido en un valor agregado para el desarrollo de las organizaciones" (p.1), sin embargo, no siempre es fácil que los programas ofertados en el mercado logren cumplir con las necesidades específicas que vayan acordes, primero con los tiempos y desplazamientos de los colaboradores y en mayor proporción, con la estrategia del negocio.

El rol y finalidad de la capacitación es buscar la mejora de las habilidades profesionales del personal estableciendo un sistema para el desarrollo profesional, garantizando que el personal adquiera la adaptación y la ejecución requeridas por el ajuste estratégico de la organización, y luego proporcionar recursos humanos adecuados e integrados para el largo plazo de la Compañía. desarrollo de plazo.

Shaun (2012) señaló que la capacitación es "una acción sistemática, planeada y permanente cuya intención es preparar, desarrollar e integrar a los recursos humanos al proceso operativo de la empresa, a través de la entrega de conocimientos, desarrollo de habilidades y actitudes necesarias" (p. 31), la finalidad de las capacitaciones es mejorar el desempeño de los colaboradores en sus actuales y futuros cargos y adaptarlos a las exigencias cambiantes del entorno. Por lo tanto, para lograr establecer una adecuada capacitación también es elemental recurrir a técnicas modernas de entrenamiento y una de ellas es el coaching, la cual permitirá establecer mediciones cuantitativas como es la generación de rentabilidad que debe impactar en las empresas (La Fortune, 2018).

Analizando de manera más profunda la capacitación, se ha podido determinar que presenta diversos significados. Algunos especialistas consideran que es un medio para 
desarrollar la fuerza de trabajo de las organizaciones; otros la interpretan más ampliamente y consideran que la capacitación sirve para un debido desempeño del puesto; asimismo, extienden el concepto a un nivel intelectual por medio de la educación general. También otros autores como Robbins (2012) se refiere a un área genérica, llamada desarrollo, que dividen en educación y capacitación: la capacitación significa preparar a la persona para el puesto, mientras que el propósito de la educación es preparar a la persona para el ambiente dentro o fuera de su trabajo (Chiavenato I. , 2017). Confrontando lo dicho anteriormente, la capacitación es el proceso educativo de corto plazo, aplicado de manera sistemática y organizada, por medio del cual las personas adquieren conocimientos, desarrollan habilidades y competencias en función de objetivos definidos En el Informe Empresa SM (2017) se conceptualiza a la capacitación como "la adquisición de conocimientos técnicos, teóricos y prácticos que van a contribuir al desarrollo del individuo en el desempeño de una actividad" (p. 12). También se sostiene como el conjunto de procesos instituidos dirigidos a prolongar y perfeccionar la educación mediante la generación de conocimientos, el desarrollo de habilidades y el cambio de actitudes, con el fin de incrementar la capacidad individual y colectiva para contribuir al cumplimiento de la misión de la organización, a la mejor prestación de servicios, al eficaz desempeño del cargo y al desarrollo personal integral. Esta definición comprende los procesos de formación, entrenamiento, adiestramiento y concientización; y según Escobar Cazal \& Escobar Reyes, (2014) "la carencia del liderazgo gerencial se debe a la falta de conocimientos en áreas y temas organizacionales, que son indispensables para el buen funcionamiento de una organización" (p. 24). En esta condición, el aún existente conformismo y la mediana acogida hacia procesos de acompañamiento en capacitación y asesorías. En efecto, estamos de acuerdo con lo indicado por Escobar toda vez que es necesario capacitarse de manera constante para poder ejercer el liderazgo.

\section{ESTRATEGIAS METODOLÓGICAS O MATERIALES Y MÉTODOS}

Tomando el diseño de investigación propuesto por Hernández Sampieri (2014); la presente investigación se abordó desde un enfoque de tipo cualitativo; porque lo que se buscó fue entender como la implementación de un programa de capacitación mejora la capacidad productiva de los recursos humanos, es decir, en su contexto natural organizacional. Por ello se analizaron a los diferentes actores o colaboradores como, 
personal administrativo, técnicos, operarios y el personal involucrado con la gestión del talento de la empresa Contratista de Minería y Construcción de Lima.

El modelo de investigación plantea que la aplicación del diseño de un plan de capacitación puede influenciar positivamente en el rendimiento y efectividad de los recursos humanos de la empresa en mención.

Tipo de investigación. Según el enfoque de investigación, es de naturaleza cualitativa y descriptiva. Por ello, la investigación se basó en la teoría fundamentada la que permitió analizar el tema de investigación desde su contexto natural, mediante el acopio de información, a través de las entrevistas a profundidad, y a partir de ello comprobar el objetivo.

Según Ruiz (2013) los planteamientos de investigación cualitativa presentan las siguientes características: énfasis en estudiar los fenómenos sociales en el propio entorno natural que ocurren; también se toma en cuenta la primacía de los aspectos subjetivos de la conducta humana sobre las características objetivas, la exploración del significado del actor. También se toma en cuenta la predilección de la observación, la guía de entrevistas semiestructurada y de profundidad como herramientas de exploración; y, el empleo del en la fase de la entrevista desglosada en el antes, durante y después de la entrevista. La estrategia de investigación de este estudio se apoyó en el método de entrevista, según indica Taylor (1987) en la entrevista el entrevistador debe de asegurar que los resultados sean comparables. El entrevistador sirve como un cuidadoso recolector de datos; su rol incluye el trabajo de lograr que los sujetos se relajen lo bastante como para responder por completo a la serie predefinida de preguntas.

El sustento de lo mencionado se sostiene en la teoría fundamentada, de acuerdo con Hernández, Fernández, \& Baptista (2014) es un diseño y un producto donde el investigador produce una explicación general o teoría respecto al fenómeno, proceso, acción o interacciones que se aplican a un contexto concreto y desde la perspectiva de diversos participantes.

\section{RESULTADOS Y DISCUSIÓN}

La propuesta y aplicación de un diagnóstico acerca de la capacitación a los colaboradores de la empresa Contratista de Minería y Construcción de Lima se desarrolla bajo un contexto de fortalecimiento del área de recursos humano, ello nos permitiría analizar las 
competencias y estrategias que viene aplicando la empresa para mejorar la gestión del talento humano.

Breve historia de la empresa SMCG. La empresa Contratista de Minería y Construcción en Lima se formó por escritura pública de fecha 7 de noviembre de 1990, concedida ante el notario público de Lima, doctor Jorge Velarde Sussoni, año en que los constituyentes resolvieron apostar por el desarrollo del país y establecieron sociedades especialistas en servicios mineros.

A lo largo de los años, la sociedad ha rescatado un rol transcendental en el perfeccionamiento de los primordiales proyectos de minería y construcción del Perú.

Desde los inicios se determinó por forjar una agraciada organización, conducente en la seguridad de los trabajadores, el respeto al medio ambiente y el desarrollo del talento mediante la aplicación de los valores, tanto en el ámbito profesional como personal.

Durante los últimos años ha diseñado soluciones integrales para sus clientes y ha consolidado la filosofía de la empresa, con la que ha crecido los últimos 25 años. En la última década, la empresa Contratista de Minería y Construcción en Lima ha crecido a una tasa anual compuesta de $19.5 \%$.

Gestionar los Recursos Humanos Condicionalmente. La manera exacta en que las prácticas de gestión de recursos humanos en la empresa Contratista de Minería y Construcción de Lima parte de la aplicación de su misión, y, ella depende de la organización y el mercado al que se enfrenta. Por ejemplo, un contratista donde los clientes frecuentemente prefieren rapidez y eficacia. En otras palabras, los sistemas de selección, formación y retribución deberían tratar de producir empleados que trabajen rápidamente y que se identifiquen con el cliente corporativo o empresarial al cual se está atendiendo y la retribución de ese personal será de una manera coherente con lo que se le exige.

Gestionar los Recursos Humanos científicamente. El éxito del movimiento de la gestión de la calidad total ha servido para darse cuenta de que la calidad surge de la información de calidad, sobre todo en lo relativo a las expectativas del cliente y a los niveles de satisfacción. Son los clientes los que definen la calidad.

En el mismo sentido, las prácticas de gestión de recursos humanos como la contratación y la retribución en la empresa Contratista de Minería y Construcción de Lima se validan en comparación con los criterios del cliente, como sus impresiones de la calidad de 
atención en las obras, lo mencionado se convierte en el Know How para la empresa en estudio.

Así mismo, a la hora de evaluar la eficacia de la gestión de los recursos humanos, las empresas deberían prestar atención a criterios relativos a los empleados (como la satisfacción en el trabajo o la rotación de los trabajadores), y también al hecho de que estas prácticas ayuden o no a conseguir satisfacer a los clientes.

En el caso de la empresa Contratista de Minería y Construcción de Lima son los gerentes y los directivos, los que determinan que prácticas de gestión de recursos humanos tienen mayores efectos sobre las estrategias de la gestión de los recursos humanos.

Gestionar los Recursos Humanos Inter-funcionalmente. Por lo general, es la organización la que debe gestionar prácticas y cuestiones diferentes para formular una estrategia que ofrezca resultados positivos en la empresa Contratista de Minería y Construcción de Lima. Es decir, existen variables importantes que influyen en el comportamiento y actitudes de los colaboradores frente a las demandas de los clientes.

La aplicación de cualquier programa de formación en la empresa debe ser consecuencia de un análisis previo de necesidades formativas, debido que la formación se desarrolla sobre determinados objetivos que se desean cubrir, tal es el caso de la empresa Contratista de Minería y Construcción de Lima. Para la implementación y propuesta del plan de capacitación se han tomado en cuenta ciertos factores para el desarrollo de la formación:

- Cambios en la organización.

- Cambios de métodos y proceso de trabajo.

- Utilización de nuevas tecnologías.

- Desaprovechamiento de las múltiples posibilidades que en ocasiones ofrece un producto.

- Aumento de los servicios que se ofrecen.

- Apertura a otros mercados.

- Admisión de nuevos empleados.

- Reducción de plantilla.

- Aumento de la competencia.

Descripción del proceso actual. Si bien en la actualidad, concurren lineamientos para tramitar el proceso de capacitación definido y formado en las políticas que se representarán en el siguiente apartado, no se ha definido un asunto claro y conciso que la 
organización conozca y pueda seguir cada vez que requiera de una actividad de capacitación.

Actualmente el Jefe de Gestión de Talento en conjunto con el Coordinador de Capacitación y Desarrollo ejecutan el diagnóstico de necesidades de capacitación mediante entrevistas con los líderes de cada área, este procedimiento se realiza entre los meses de octubre y enero. Una vez levantadas las necesidades, se priorizan y se someten a aprobación de la Gerencia General.

Por otro lado, las necesidades aprobadas se plasman en el plan anual de capacitación para últimamente elaborar el programa de capacitación que es un documento donde las necesidades aprobadas se ponen en un cronograma anual. Este cronograma es utilizado por el área de Capacitación y Desarrollo para realizar el seguimiento de la ejecución de las actividades y del presupuesto planificado para el año.

En caso, algún área demande alguna capacitación que no se encuentre inscrita en el plan de capacitación anual, este nuevo requerimiento se someterá a la aprobación de la gerencia de Capital Humano.

Como soporte al proceso antes descrito, se han elaborado algunos formatos que se referirán en el siguiente apartado, sin embargo, estos formatos no son utilizados por todos los implicados en el proceso, por lo que no se cuenta con registros completos que puedan sostener el proceso en su totalidad.

Políticas asociadas. Las políticas perfeccionadas con el fin de proponer soporte al proceso de formación y desarrollo de la empresa Contratista de Minería y Construcción de Lima son el Procedimiento de Capacitación de personal y la Directiva de Capacitación de Personal, en ellas sobresalen las siguientes definiciones que consideramos oportuno detallar:

- Capacitación: Es la adquisición de conocimientos técnicos, teóricos y prácticos que van a contribuir al desarrollo del individuo en el desempeño de una actividad. Se entiende por capacitación el conjunto de procesos organizados dirigidos a prolongar y a complementar la educación inicial mediante la generación de conocimientos, el desarrollo de habilidades y el cambio de actitudes, con el fin de incrementar la capacidad individual y colectiva para contribuir al cumplimiento de la misión institucional, a la mejor prestación de servicios, al eficaz cometido del cargo y al 
progreso personal integral. Esta definición comprende los procesos de formación, entrenamiento, adiestramiento y sensibilización.

- Competencia: Resulta de una combinación dinámica de atributos en relación a conocimientos, habilidades, actitudes y responsabilidades que describen los resultados del desempeño considerados idóneos en el medio laboral.

- Capacitación interna: Desarrollada en base a necesidades específicas sean estas técnicas, funcionales o de competencias personales. El contenido de la capacitación lo elabora un profesional que forme parte de la empresa y que tenga pleno conocimiento en la materia. Esta actividad se caracteriza por ser no remunerada económicamente pero que constituye un alto impacto para los beneficiarios por el conocimiento especializado que representa y que no se consigue en el mercado externo.

- Capacitación externa: Es la capacitación desarrollada por profesionales que no pertenecen a la empresa, adquirida a proveedores de servicios educativos calificados.

Propuesta del plan de capacitación. La estructura orgánica de la sociedad de Minería y Construcción de Lima, las áreas “core del negocio" son la Unidad de Negocio Minería y la Unidad de Negocio Construcción. Mientras que las áreas de Soporte del Negocio, en las que estamos enfocando el presente trabajo, son las siguientes:

- Área de Proyección Estratégico.

- Área de Desarrollo de Nuevos Negocios.

- Área Comercial.

- Área de HSE (Seguridad, Salud Ocupacional y Medio Ambiente).

- Área de Administración y finanzas.

- Área de Logística.

- Área de Capital Humano.

- Área Legal.

- Área de Equipos y Activos Fijos.

Gerencia de Capital Humano. El área de Capital Humano de la empresa Contratista de Minería y Construcción en Lima tiene el objetivo de ser socio estratégico del negocio, anticipándose a sus necesidades a través de la gestión del desarrollo de las personas y reforzando la cultura de la empresa. Para lograr este objetivo, el área de Capital Humano cuenta con las siguientes sub áreas: 
- Operaciones de Capital Humano: Su misión es asegurar el cumplimiento de los lineamientos y procesos operativos de Capital Humano, con el fin de que los proyectos/obras se desarrollen con el mínimo riesgo laboral. Dentro de esta área se llevan a cabo los procesos de:

- Administración de personal.

- Gestión del bienestar social.

- Prevención de riesgos laborales.

- Capital humano en obras.

- Negociaciones sindicales.

- Gestión del Talento: Su misión es identificar al mejor talento (alineado a la cultura organizacional) y desarrollarlo con la finalidad de que pueda asumir los futuros cambios organizacionales requeridos para que la empresa pueda mejorar su competitividad en el mercado. Los procesos gestionados por esta área son:

- Capacitación y desarrollo.

- Cultura organizacional.

- Estructura orgánica.

- Clima laboral.

- Atracción de Talento: Su misión es proponer y atraer talento para cumplir con la cobertura de vacantes con el perfil idóneo según las necesidades de las áreas y/o proyectos a través de un proceso de reclutamiento y selección efectivo. Los procesos que tiene esta área bajo su cargo son:

- Planeamiento de personal.

- Búsqueda y dotación de personal.

- Personal clave - licitaciones.

- Elaboración y actualización de perfiles de puesto.

- Compensaciones: tiene como misión contribuir a la atracción y retención del talento a través de estrategias remunerativas y de beneficios competitivos alineados a la estrategia de la empresa, para ellos, cuenta con los siguientes procesos:

- Diseño y aplicación de la estructura salarial.

- Proceso de incrementos anuales.

- Diseño de planes de beneficios.

- Presupuesto de personal anual. 
- Generación de información técnica para negociaciones sindicales.

- Comunicación Interna: tiene como misión comunicar, alinear, motivar y comprometer a los colaboradores con la identidad de la organización, a través de la gestión de una comunicación interna estratégica que cree valor y refuerce el sentido de pertenencia.

En la Figura 1 se presenta la estructura organizacional del área de Capital Humano.

Figura 1 Organigrama del área de Capital Humano

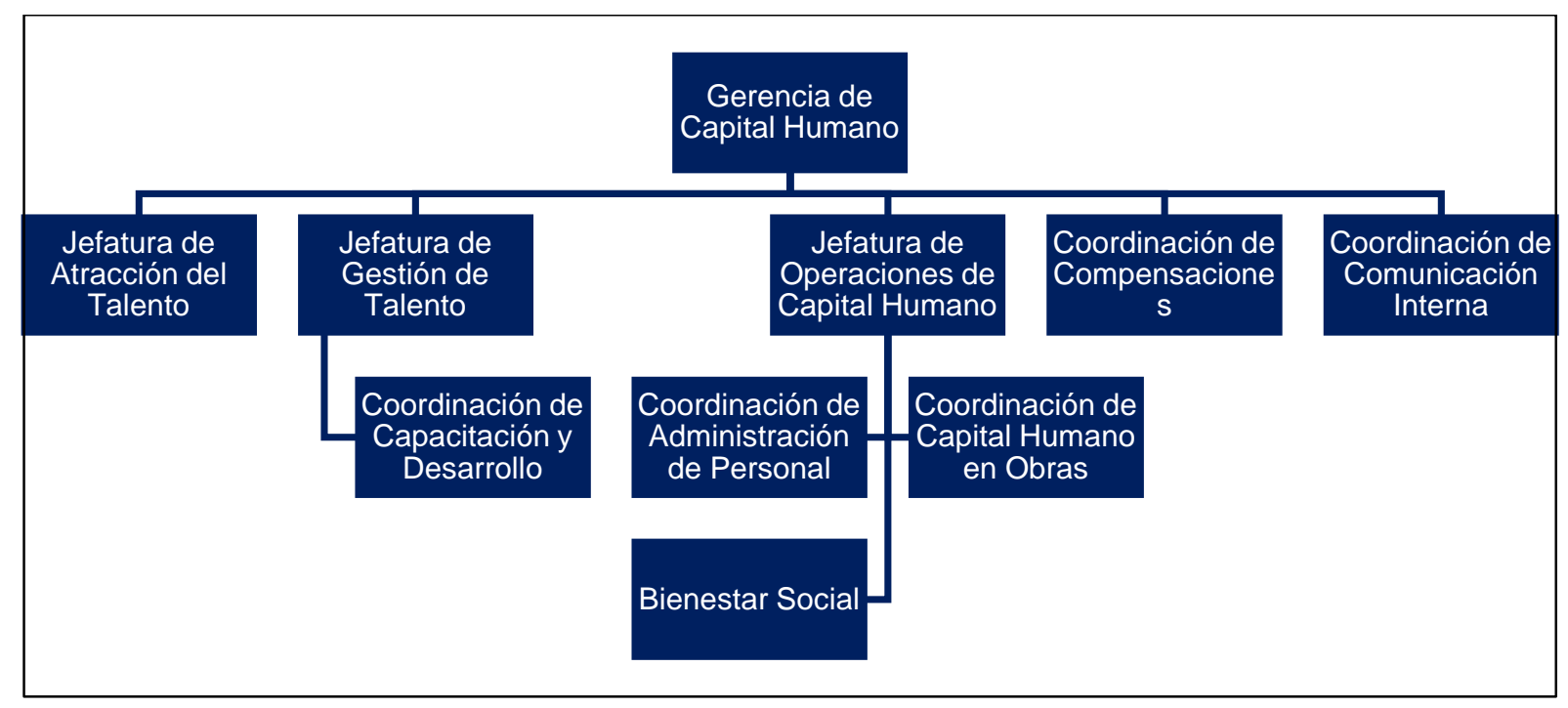

\section{Proceso de capacitación y desarrollo}

En la estructura organizacional del área de Capital Humano, el proceso de capacitación y desarrollo se encuentra ubicada dentro de la sub área de Gestión de Talento, como se muestra a continuación en la Figura 2.

Figura 2. Organigrama de la sub área de Gestión del Talento

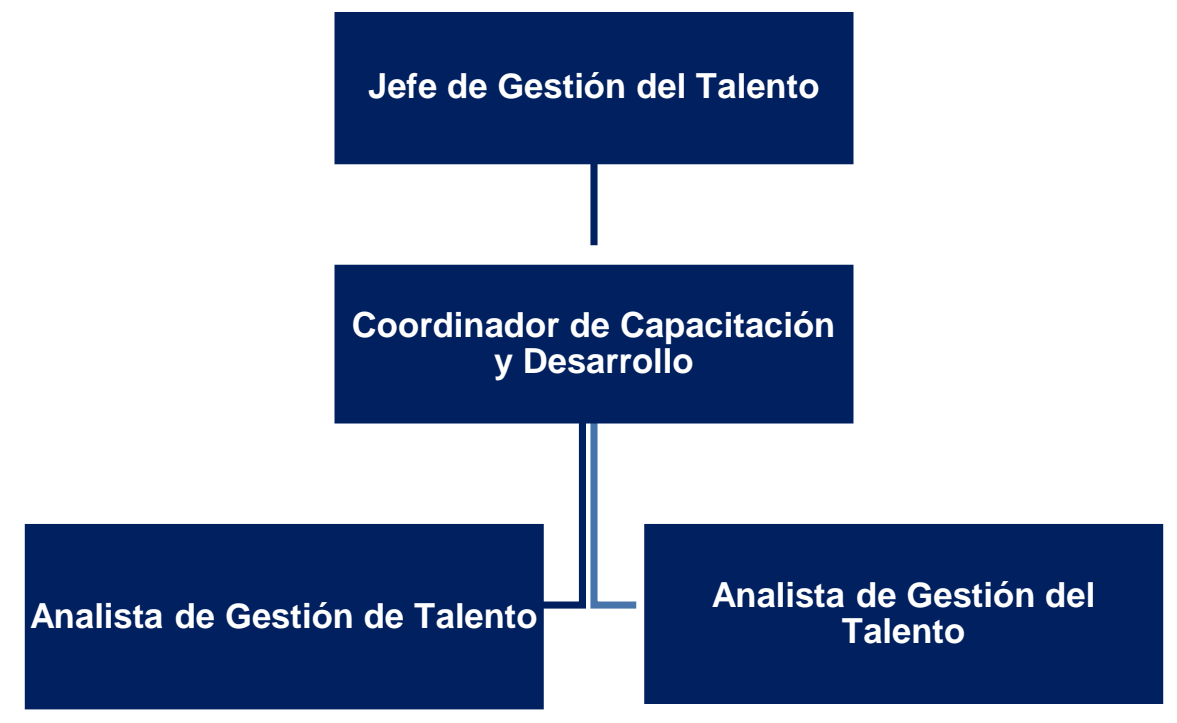


Dentro de las actividades más resaltantes que se llevan a cabo en el área de capacitación y desarrollo se encuentran:

- Detección, desarrollo y retención del talento.

- Gestión de las capacitaciones.

- Evaluaciones de competencias.

- Promociones.

- Líneas de sucesión.

\section{DISCUSIÓN}

Pinto (2014) señaló que el papel que desempeña la capacitación consiste en "permitir que la organización logre sus objetivos estratégicos cada vez con mayor productividad y eficiencia, ampliando asimismo la cantidad de talento y la capacidad disponible" (p.78). En efecto, la importancia de los procesos de capacitación radica en una excelente oportunidad para expandir la base de conocimiento de todos los empleados, pero muchos empleadores encuentran costosas las oportunidades de desarrollo. Los empleados también invierten su tiempo de trabajo mientras asisten a las sesiones de capacitación. A pesar de los posibles inconvenientes, la capacitación y el desarrollo proporcionan a la empresa en su conjunto y a los empleados individuales beneficios que hacen que el costo y el tiempo sean una inversión recuperable.

En la actualidad la capacitación es la respuesta a la necesidad que tienen las empresas de contar con un personal calificado y productivo. Para las organizaciones la capacitación es de vital importancia porque contribuye al desarrollo personal y profesional de sus colaboradores que a su vez redunda en beneficios para la empresa. En opinión de Ulrich citado por Díaz (2014), el sentido de pertenencia y compromiso de los trabajadores "garantiza el éxito de cada empresa y siempre que se hable de talento humano se debe partir de un proceso enfocado en resultados, al involucrar aspectos estratégicos y la formación y capacitación del talento humano" (p. 14).

Una de las razones por las que las empresas capacitan a su personal radica en la obsolescencia, de esta forma se procura actualizar los conocimientos con las nuevas técnicas y métodos de trabajo que garantizan eficiencia. Promover la constante actualización y capacitación de los colaboradores permite innovar en los procesos, procedimientos y forma de hacer las cosas, desarrolla capacidades y destrezas que se convierten en activos intangibles de gran valor para la empresa (Rodriguez, Granados, \& 
Velandia, 2014).

Otro de los aspectos que fundamentan la importancia de la capacitación, es que representa una oportunidad para expandir la base de conocimiento de todos los colaboradores de la empresa. Por lo tanto, proporciona a la empresa y a los empleados, beneficios que hacen que el costo y el tiempo sean una inversión que se refleje en resultados positivos para el talento humano.

Un programa de capacitación fortalece las capacidades que cada empleado necesita para mejorar su productividad o rendimiento, de esta manera, la capacitación lleva a los empleados a un nivel superior para que tengan habilidades y conocimientos homogéneos y permite que puedan hacerse cargo de las funciones que les corresponden, sin ayuda y supervisión constante. Además, la capacitación es considerada una de las estrategias para el desarrollo de los empleados en el contexto de los proyectos o del desempeño propiamente que se le encomiende (Sánchez \& Jiménez, 2015).

Otro de los elementos también es abordar las debilidades, la mayoría de los empleados tienen algunas debilidades en sus habilidades laborales. Un programa de capacitación le permitiría fortalecer las habilidades que cada empleado necesita para mejorar. Un programa de desarrollo lleva a todos los empleados a un nivel superior para que todos tengan habilidades y conocimientos similares. Esto ayuda a reducir los vínculos débiles dentro de la empresa que dependen en gran medida de los demás para completar tareas básicas de trabajo (Sánchez \& Jiménez, 2015). Proporcionar la capacitación necesaria crea un personal con conocimientos generales con empleados que pueden hacerse cargo de los demás según sea necesario, trabajar en equipos o trabajar de forma independiente sin la ayuda constante y la supervisión de los demás.

Otro elemento de importancia es alcanzar el rendimiento de los empleados, un empleado que recibe la capacitación necesaria está en mejores condiciones para realizar su trabajo. Se vuelve más consciente de las prácticas de seguridad y los procedimientos adecuados para las tareas básicas (Cros, 2016). La capacitación también puede aumentar la confianza del empleado porque tiene una mejor comprensión de la industria y las responsabilidades de su trabajo. Esta confianza puede impulsarla a desempeñarse aún mejor y pensar en nuevas ideas que la ayuden a sobresalir.

La capacitación constante también mantiene a sus empleados a la vanguardia de los desarrollos de la industria. Los empleados que son competentes y se encuentran a la 
vanguardia de los cambiantes estándares de la industria ayudan a su empresa a ocupar un puesto como líder y fuerte competidor dentro de la organización.

En relación con la importancia que debe tener un programa o plan de capacitación es la consistencia, un programa estructurado de entrenamiento y desarrollo asegura que los empleados tengan una experiencia consistente y conocimiento previo. La consistencia es particularmente relevante para las políticas y procedimientos básicos de la compañía. Todos los empleados deben conocer las expectativas y los procedimientos dentro de la empresa. Esto incluye seguridad, discriminación y tareas administrativas. Poner a todos los empleados a través de capacitación regular en estas áreas asegura que todos los miembros del personal al menos tengan exposición a la información (Chiavenato I. , 2011).

Por otro lado, para efectos externos de la empresa, se tiene la satisfacción del empleado. Los empleados con acceso a programas de capacitación y desarrollo tienen la ventaja sobre los empleados de otras compañías que solo tienen oportunidades de capacitación por su cuenta. La inversión en capacitación que hace una empresa muestra a los empleados que se valoran. La capacitación crea un lugar de trabajo de apoyo, los empleados pueden obtener acceso a una capacitación que de otro modo no conocerían ni buscarían por sí mismos (Chiavenato I. , 2017). También se sienten apreciados y desafiados a través de las oportunidades de capacitación y pueden sentir una mayor satisfacción hacia el desarrollo del trabajo.

\section{CONCLUSIÓN O CONSIDERACIONES FINALES}

- Se concluye que el área de recursos humanos de la empresa es una de las más complejas del medio empresarial, esta complejidad se debe a la amplitud de sus unidades de negocios y a la cobertura que tiene a nivel nacional e internacional.

- Los resultados que la empresa ha logrado durante estos últimos años, se deben elementalmente a la gestión de los recursos humanos, liderados por su gerente general; quedando demostrado que la experiencia y la solidez se debe al manejo de la dirección empresarial.

- La estructura horizontal que tiene la empresa funciona adecuadamente, como se puede apreciar en los anexos, cada gerencia cuenta con un organigrama y su respectivo manual de funciones articulado a las políticas y objetivos de la empresa.

- Si bien es cierto que la gestión de recursos humanos de la empresa es una de las más 
sólidas debido a los resultados que muestra, es recomendable que se implementen y complementen estrategias como la gestión por competencias, ya que esta herramienta tiene mayor amplitud en su enfoque, ya que analiza una serie de características más precisas en cuanto a las conductas, actitudes, habilidades, conocimientos, etc. de cada individuo, lo cual permite, no sólo una mejor definición del perfil de competencias del puesto sino la posterior evaluación en el desempeño individual.

\section{La propuesta está conformada por los siguientes lineamientos:}

- Trabajar un proceso de capacitación basado en el plan estratégico de la empresa.

- Establecer lineamientos claros, para ello se trabajará una política de capacitación y otra política de beneficios educativos.

- Las áreas de SSOMA y Equipos seguirán gestionando sus capacitaciones, sin embargo, el área de capacitación - Capital humano consolidará la información de capacitación total de la empresa por lo cual SSOMA y equipos deben reportar esta información a Capital humano de manera mensual para poder tener indicadores reales de capacitación.

- El rol del área de capacitación será el de planificar, ejecutar y dar seguimiento a las capacitaciones de la empresa. Además, será un asesor de las diferentes áreas de la empresa en cuanto a la detección de necesidades, y conveniencia de la realización de capacitaciones.

Figura 3. Organigrama de la sub área de Gestión del Talento

\section{Plan Estratégico SMCG}

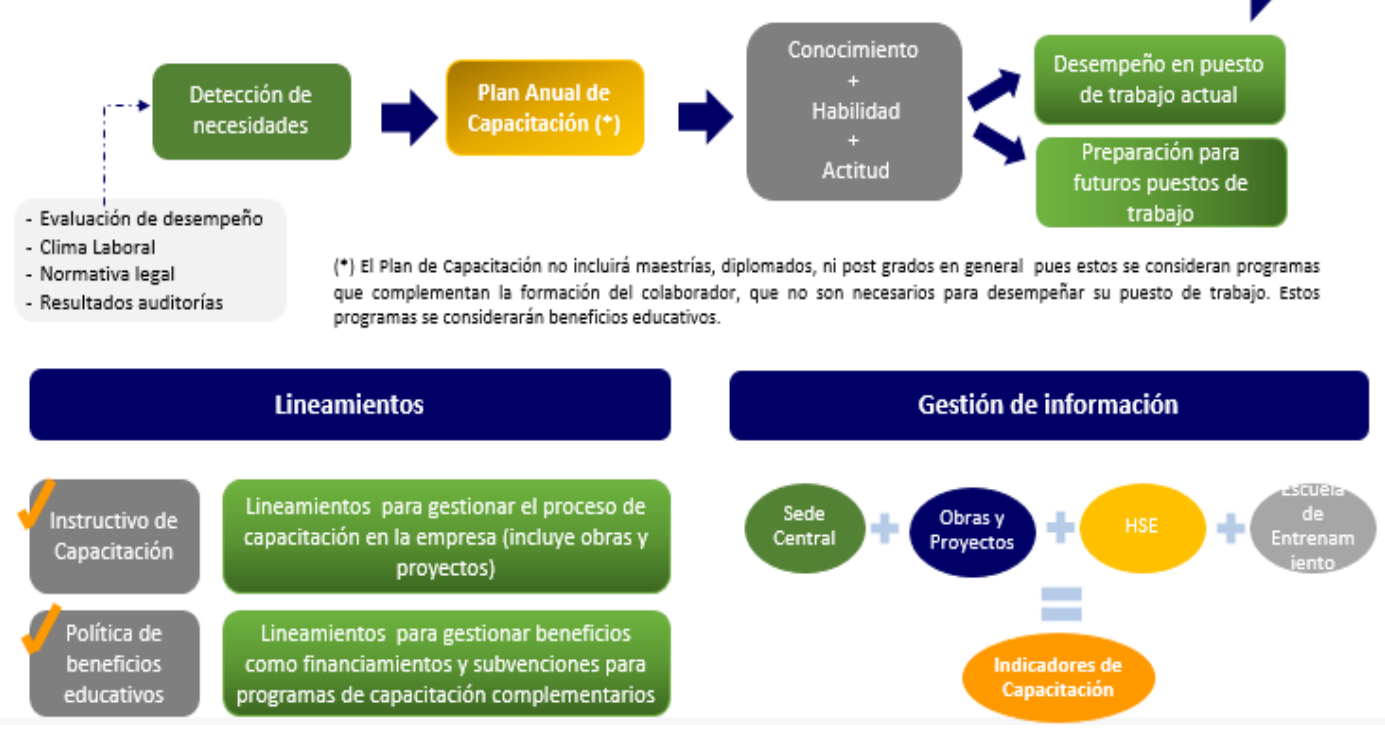




\section{Estrategia de capacitación en SMCG}

La capacitación y el desarrollo de los empleados es un término amplio que abarca múltiples tipos de aprendizaje de los empleados. La estrategia que se aplicará estará de acuerdo a la capacitación efectiva como una necesidad comercial que ayude a los miembros del equipo a desarrollar las habilidades necesarias en su puesto de trabajo.

Dentro de las mediciones, la capacitación ayudará a los empleados a dominar nuevos procedimientos y reforzar| los protocolos existentes. Cuando los miembros del equipo son consistentes, la administración está mejor equipada para evaluar e identificar los problemas subyacentes en todas las operaciones. Toda empresa necesitará identificar sus propias necesidades internas para desarrollar una estrategia de capacitación efectiva.

\section{LISTA DE REFERENCIAS}

Barney, J., \& Wrigth, P. (1998). On Becoming a strategic Parther: The role of Human resources in gaining competitive advantage. Human Resource Management, , 3146.

Cappelli, P; Singh, U. (2014). Distinctive hurnan resources are flITIl's core cornpetencies. Ecuador: Investigaciones Europeas de Dirección y Economía de la Empresa.

Chiavenato, I. (2011). Gestión del Talento Humano. México: McGraw-Hill.

Chiavenato, I. (2017). Administración de recursos humanos. El capital humano de las organizaciones. México: McGraw-Hill.

Conexionesan. (18 de Junio de 2016). Cinco pasos del plan de capacitación. Recuperado el 21 de marzo de 2018, de https://www.esan.edu.pe/apuntesempresariales/2016/07/cinco-pasos-del-plan-de-capacitacion/

Cros, J. (2016). Capacitación yapos; en casayapos; con muchas ventajas. Business And Economics--Economic Situation And Conditions, 45.

De Cenzo, R. (2011). Administración de Recursos Humanos. México: Limusa.

Deloitte Consulting LLP, \& Deloitte, B. (2014). Tendencias globales del capital humano 2014: Involucrando a la fuerza laboral del siglo 21. Paris: About Deloitte University Press.

Dessler, G., \& Varela, R. (2011). Administración de Recursos Humanos. México: Enfoque Latinoamericano. 
Díaz Brochet, N. (2015). La gerencia de recursos humanos debe agregar valor. PricewaterhouseCoopers, 12-46.

Díaz, N. (2014). La gerencia de recursos humanos debe agregar valor. Consultoria PricewaterhouseCoopers, 45.

EDWPUE. (30 de Noviembre de 2016). Capacitación yapos; en casayapos; , con muchas ventajas. Portafolio; Bogotá, 13.

Escobar Cazal, E., \& Escobar Reyes, G. (2014). Diagnóstico y capacitación a entidades sin ánimo de lucro. Provincia del Sumapaz, Colombia. Pensamiento y gestión, 43.

Hernández, O., \& Jáuregui, K. (2015). A snapshot of training practices in Peru. Business School, Instituto Tecnológico y de Estudios Superiores de Monterre, 67-85.

Informe Empresa SM. (2017). Directiva para la capacitación del personal. Lima.

La Fortune. (2018). Capacitación de recursos humanos ofrece rentabilidad. Business And Economics--Economic Situation And Conditions, 34.

Lodoño, C. (2013). Capacitación y desarrollo. Lima: Revista Universidad EAFIT.

Macías, C., \& Martínez, A. (2012). Contribución de la gestión de recursos humanos a la gestión del conocimiento. Estudios Gerenciales, 133-148.

Méndez, E. (12 de Setiembre de 2004). El Diagnóstico de Necesidades de Capacitación es un Asunto Local. Recuperado el 12 de Marzo de 2018, de Revistas de Ciencias Administrativas y Financieras de la Seguridad Social: http://www.scielo.sa.cr/scielo.php?script=sci_arttext\&pid=S140912592004000100003

Noe, R. (2012). Employee Training \& Deve Development. New York: McGraw Hill. Ojeda, J. (2018). Gestión del talento Humano.. Lima.

Ospina, H. (13 de Enero de 2017). Nuevos paradigmas en Gestión Humana. Revista Ciencias Estratégicas, 18(23), 79-97.

Pérez, M., Prieto, I., \& Sierra, C. (2014). Gestionando el conocimiento a través de la gestión de recursos humanos: análisis empírico en el sector de automoción. Academia, Revista Latinoamericana de Administración, 34-56.

Pinto, R. (2014). Planeación estratégica de capacitación. México: Trillas.

Robbins, S. (2012). Comportamiento Organizacional. México: McGraw-Hill. 
Rodríguez, D., Granados, P., \& Velandia, S. (2014). Prácticas de Gestión Humana en empresas del sector servicios: hoteles, bares y restaurantes de la ciudad de Tunja. Universidad Pedagógica y Tecnológica de Colombia, 43.56.

Sánchez, Y., \& Jiménez, P. (2015). Percepciones del impacto de la capacitación, compensación y selección del personal en la eficiencia de los proyectos. $A D$ minister $N^{o}$. 27, 5-26.

Shaun, A. (2012). Administración de personal (Novena ed.). México: Trillas.

SMCG. (2018). Directiva de capacitación de personal. Lima.

Truelove, S. (2014). The handbook of training. Oxford Publishers: Blackwell.

Ulrich, D. (2013). Human Resources Champions. Harvard Business School Press. USA.

UNACEM. (31 de Diciembre de 2017). Reporte de sostenibilidad 2016. Obtenido de http://www.unacem.com.pe/mayrs/rs2016/reporte/

Ureña, Y., Jiménez, I., Mejía, A., \& Vilarete Molina, J. (2014). Cultura Innovativa: Prospectiva de las condiciones estratégicas para el desarrollo del capital humano. Revista Clío América, 8(16), 23-43.

Viltard, L. (21 de Enero de 2016). La configuración del mercado de Educación Superior y Capacitación Corporativa (CC). Hacia la Universidad Corporativa (UC), 1216.

Viltrad, L. (2014). La configuración del mercado de Educación Superior y Capacitación Corporativa (CC). Hacia la Universidad Corporativa, 13-32. 